\title{
THE CONTINUUM SPECTRA OF ACCRETION DISCS IN NOVALIKE OBJECTS
}

\author{
G. Shaviv \\ Dept. of Physics and Space Research Institute \\ Israel Institute of Technology \\ IL 32000 Haifa \\ R. Wehrse \\ Institut f. Theoret. Astrophysik \\ D 6900 Heidelberg
}

\section{Introduction}

The exact value of the accretion rate in novae is very important to the theory of thermonuclear runaways and nova statistics. Estimates of the typical accretion rates in nova and novalike objects are $10^{18} \mathrm{~g} / \mathrm{s}\left(1.610^{-8} \mathrm{M}_{\odot} / \mathrm{yr}\right)$. Patterson (1984) estimated that if the above high accretion rate prevails the expected number of novae according to Bath and Shaviv (1976) exceeds the observed number. Prialnik, Livio, Shaviv and Kovetz (1982) have shown that no thermonuclear runaway can be obtained if the accretion rate is as high as few $10^{-9} \mathrm{M}_{\odot} / \mathrm{yr}$. Actually, the very strong thermonuclear runaways (TNRs) are obtained only for very low accretion rates namely, $10^{-10}$ to $10^{-11} \mathrm{M}_{\odot} / \mathrm{yr}$. A way out from this inconsistency was suggested by Shara, Livio, Moffat and Orio (1986) in the form of the hibernation model. In this model the high accretion rate is found only shortly before and soon after the nuclear runaway and the nova spends most of the time in a state of very low accretion rate - so low that most novae are not observed at all. The high accretion rate, according to this theory, is a precursor to the runaway. The hibernation model is very attractive in spite of a meager observational proof so far and theoretical justifications. Yet, it is one of the most promising ideas in this field. The hibernation scenario claims that the accretion rate is a function of time. It is high just prior to the runaway and it continues to be high for some time just after the runaway. It then declines to very low values. From the point of the model for the thermonuclear runaway the problem is how the star reacts to a temporary high accretion rate that comes after a long period of low accretion rate (Shaviv and Starrfield, 1987). Thus, even if the accretion rate is low during most of the time the phase of high accretion can still affect the outcome of the thermonuclear runaway. The crucial question from the hibernation and TNR point of view is how much mass is accreted with a very low accretion rate and how much mass is accreted during the episode of high accretion. If most of the mass is accreted at high accretion rate hibernation does not help to resolve the problems the theory of TNR has with the high accretion phase. It is therefore important to find the accurate accretion rate in nova and novalike objects. The purpose of this paper is to discuss the accretion rates in novalike objects. There are several observational results for novalike objects that allow us today to evaluate quite accurately the accretion rates from the continuum energy distribution of the accretion disc.

The organization of this paper is as follows: since the meeting is dedicated to novae and not to accretion discs and the time alloted to each speaker is short, we will first present the results and the implications and leave the discussion to the end. 


\section{Velorum}

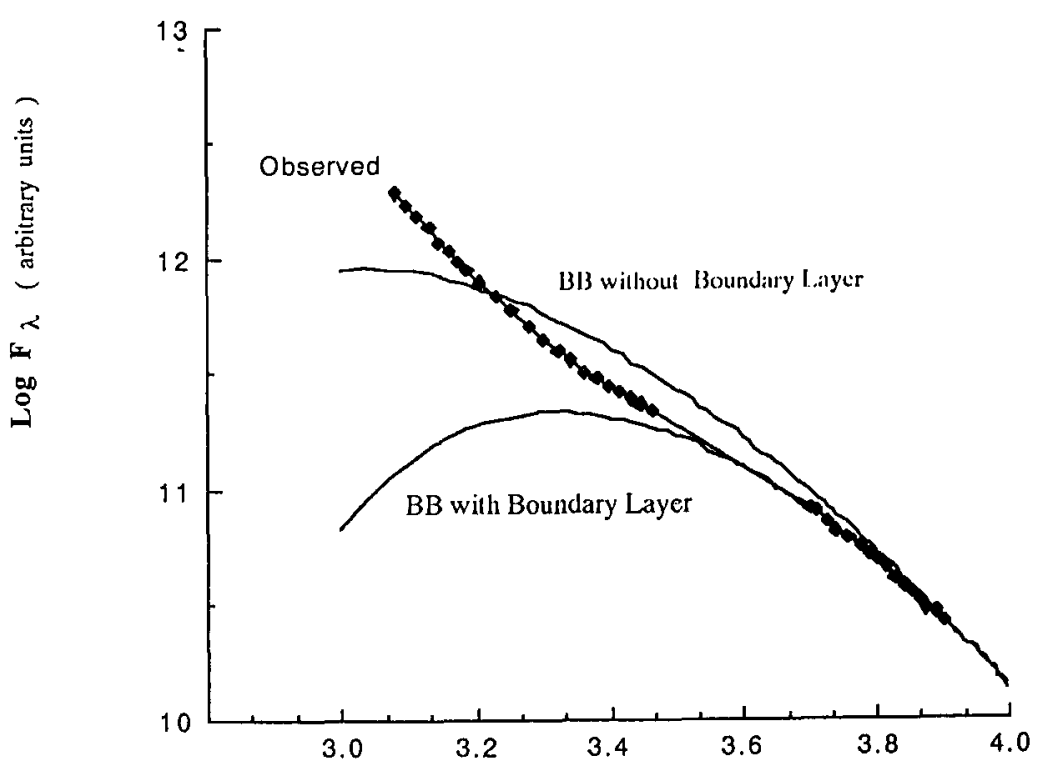

$\log \lambda$

Figure 1 The comparison between the observed energy distribution from IX Vel and the predicted one assuming the disc to radiate like a collection of black bodies. The theoretical model is evaluated with and without a boundary layer between the disc and the star: The model of the boundary layer assumed here is that of Tylenda and the boundary layer radiates like a black body. The data are form Haug.

\section{The Need for a Detailed Model}

The simplest assumption is that the disc radiates like a collection of black bodies. Under this assumption each ring of the disc radiates like a black body with the proper effective temperature. The effective temperature is determined by the luminosity and this in turn is determined by the accretion rate. Hence if the latter is assumed, the run of the effective temperature as a function of radius is known and if one adds to it the assumption that each radial ring radiates like a black body (with the proper effective temperature) then the emitted energy distribution can easily be found. We compare in Fig. 1 the results obtained under this assumption with the observations of the system IX Vel ( Haug, 1987). The assumed accretion rate is $10^{18} \mathrm{~g} / \mathrm{s}$. As can be seen, the 
comparison is quite bad irrespective of what we assume for the boundary layer. The fit in this case was such that the fluxes agree in the IR regime. Recall that about half of the total accretion luminosity is emitted by the boundary layer. There is no way to fit the UV by means of black bodies since the slope is so different.

The inadequacy of the black body is obvious. It is instructive to see the differences between the theoretical flux obtained from the detailed model used here to derive the accretion rates and the predictions of the black body model. These are shown in Fig. la calculated again for an accretion rate of $10^{18} \mathrm{~g} / \mathrm{s}$.

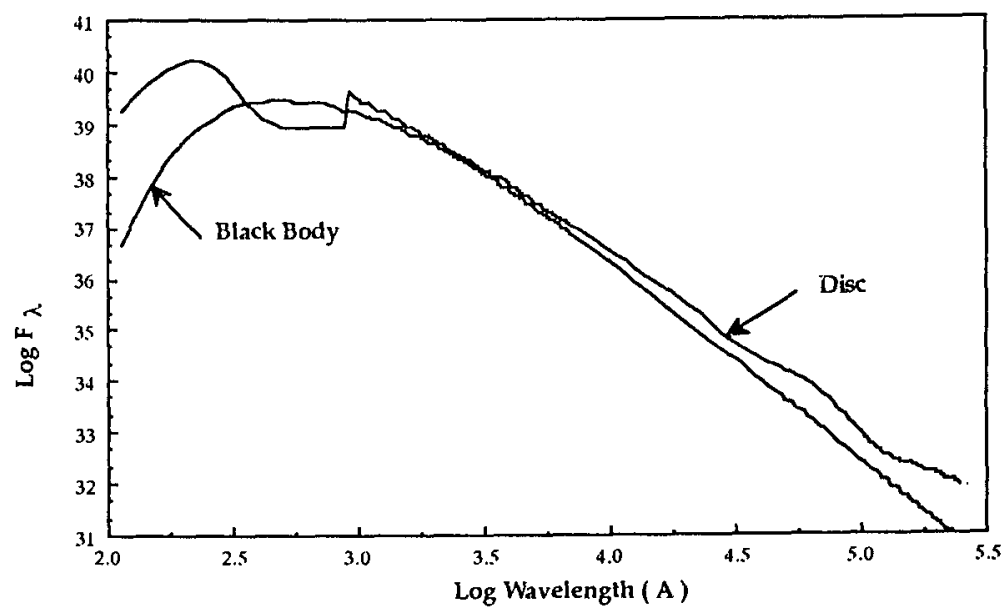

Figure 1a $A$ comparison between the energy distribution assuming the disc to radiate like a black body and our model. The accretion rate is $10^{18} \mathrm{gm} / \mathrm{sec}$. The mass of the white dwarf is $0.8 \mathrm{M0}$.

We picked for demonstration the "good" case namely, the accretion rate that gives a nice fit to the visible wavelengths and we tried to fit the black body to the distribution obtained from our model in the visible range. At high accretion rates the maximum of the flux is in the far UV below the high absorption of hydrogen. The energy distribution in the UV is determined by the strong absorption edges of hydrogen and other species. The distribution in the visible is wholly determined by the redistribution of the UV radiation. Hence, the black body distribution is very misleading.

\section{Comparison with Observations and Results for the Accretion Rate}

Nova-like variables are known to have a high accretion rate. In this respect they are supposed to resemble classical novae before and after the eruption. Of particular importance in obtaining reliable rates from fitting the observed.spectra is the use of a wide range of wavelengths. The contribution of certain parts of the disc to a particular 
wavelength range may be more important than other parts. However, all the parts of the disc contribute to all wavelengths. Hence, a fit over a wide wavelength range is necessary, if accurate values for the accretion rates are desired.

The following three systems IX-Vel=CPD $48^{\circ} 1577$, V3885 SGR and RW Sex have been observed over a wide frequency range and the results have been assembled by Haug (1987). The properties of these systems are well known. A fourth system newly discovered is PHL227 which we try to fit as well.

Cataclysmic systems have few sources of radiation (both stellar photospheres, hot spot, boundary layer, accretion disc). However, when the accretion rate is so high as assumed here for novalike variables it is safe to consider the accretion disc and to ignore all other sources. Hence we examine the continuum energy distribution from these systems assuming that all the observed flux is due to the accretion disc. There is good reason to suppose that the energy emitted by some of the other sources in the system may depend on the total accretion rate. For example, the total emissivity of the hot spot is probably related to the total accretion rate. However, the hot spot is likely to contribute in the infra-red, if at all. Thus one should be prepared to see deviations in the IR. Fortunately, these are not seen in these systems and we find a good agreement over the entire observed range of frequencies. The analysis of lines and other details will be carried out elsewhere. Since we are now in the process of obtaining better expressions for the effect of the inclination on the spectra (limb darking effects in discs) we applied the simple cosine law for systems that are not seen face on.

Haug (1987) presented data of UX-UMa systems over a wide range of wavelengths. The data so collected refer to the stationary state and hence can serve to find the accretion rate in this phase. These nova-like systems were studied in great detail since 1965 and reviews are given by Wargau et al. 1983, Haug and Drechsel 1985, Greenstein and Oke 1982. To simplify the discussion here we adopt all the parameters of the systems as tabulated by Haug except for the accretion rate. We did not try at this stage to reiterate the parameters and improve the fit beyond the one obtained here. Our main purpose is to show the first order effect of the theoretical models and not to analyze the systems under consideration for their parameters. This will be done later. The relevant data for the systems is given in Table 1 . In the calculations reported here we assumed the mass of the white dwarf to be $0.8 \mathrm{M}_{\odot}$

Table 1

\begin{tabular}{lrrr} 
System & \multicolumn{2}{c}{ The Stellar Properties of the Systems } \\
& Mass $/ M_{\odot}$ & $\begin{array}{r}\text { Inclination } \\
\text { angle (degrees) }\end{array}$ & $\begin{array}{r}\text { Outer disc } \\
\text { radius }\left(10^{9} \mathrm{~cm}\right)\end{array}$ \\
RW Sex & $0.80 \pm 0.3$ & $43 \pm 11$ & $34 \pm 5$ \\
V3885 Sgr & $0.80 \pm 0.2$ & $55 \pm 5$ & $31 \pm 3$ \\
IX Vel & $0.64 \pm 0.1$ & $61 \pm 4$ & $27 \pm 2$
\end{tabular}

The data presented by Haug are composed of observations in the UV (IUE low dispersion spectra, $1200-2900 \AA$; optical spectroscopy $4000-10,000 \AA$ and the near IR data in the JHK(L) bands). Typical error of the optical and the IR photometry is estimated at $0.02 \mathrm{mag}$ and 0.05 mag respectively. 
Figures 2, 2a, and 2b present the comparison of our model with the observed energy distribution from the system RW Sex. The comparison is carried out with two accretion rates. We first show the comparison with a single model and then with the two rates together.

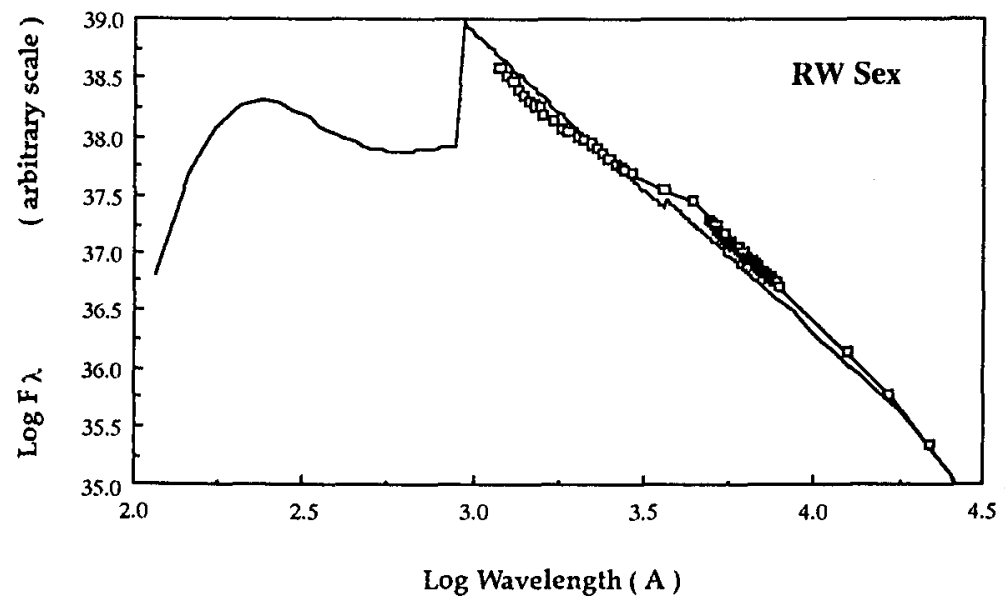

Figure 2 Comparison between the observational and theoretical energy distributions for the system RW Sex. The observational data (marked by open squares) are from Haug. The theoretical model was calculated for an accretion rate of $10^{17} \mathrm{gm} / \mathrm{sec}$.

As can be seen, an assumed accretion rate of $10^{17} \mathrm{~g} / \mathrm{s}$ produces a good agreement between the theory and the observations over the wide range of wavelengths. The high accretion rate $10^{18} \mathrm{~g} / \mathrm{s}$ does not reproduce the observed data as well.

The quality of the agreement with the observation is further appreciated when we observe a smaller range of wavelengths. For this reason we show in Fig. $2 \mathrm{~b}$ the same object compared with the same data but over a smaller range of wavelengths. Here we show the theoretical models for the two accretion rates. All other parameters are kept the same, in particular the outer radius of the disc is kept fixed. The observed distribution is copied and displaced by a constant amount so as to agree best with the theoretical curve. In other words, the theoretical curves are calculated for the same fixed (and arbitrary distance) and the observed data are shifted up and down so as to fit best the theoretical curve. Note that the calculated curves converge towards each other in the IR. IR colors are not good indicators for the accretion rates if they are high.

The particular shape of the energy distribution and the maximum near $400 \AA$ are due to several effects, the most important ones being the decrease in the opacity 


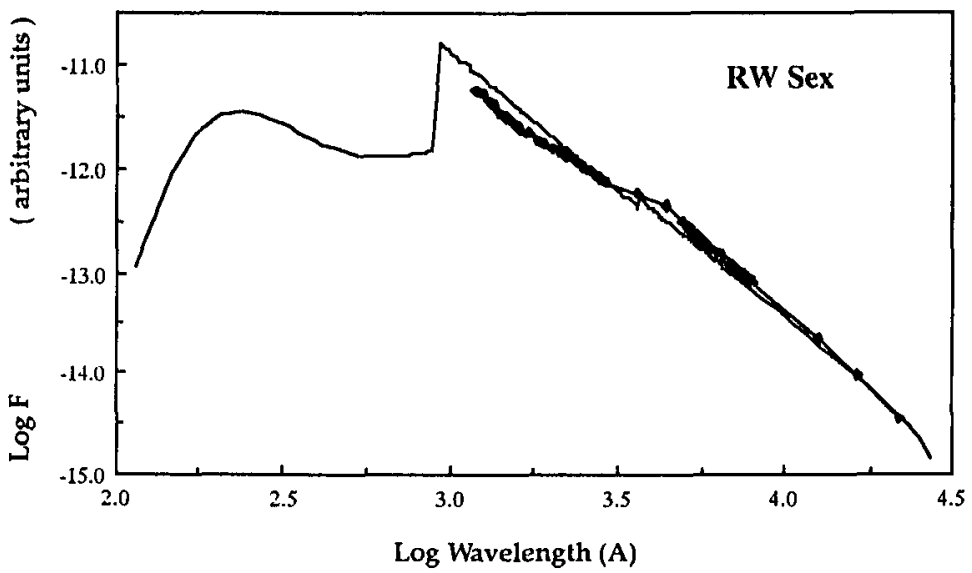

Figure 2a The same as figure 2 but the theoretical accretion rate is $10^{18} \mathrm{gm} / \mathrm{scc}$. The observational data are marked by full squares.In some regions the density of observational points is so high that they merge to form a heavy line.

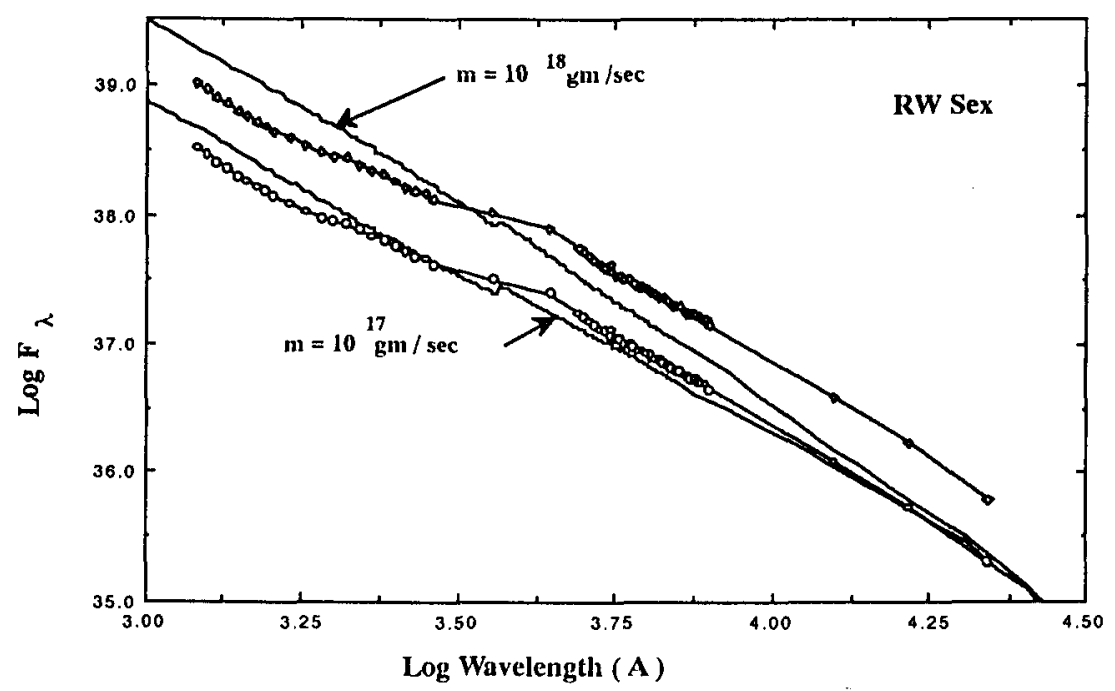

Figure $2 \mathrm{~b}$ The comparison between the two theoretical accretion rates The units of the flux are arbitrary. The observed data was copied and shified up or down so as to fit best the theory ignoring possible constraints from the distance etc. 
for wavelengths below the Lyman jump and the peak in the black body distribution corresponding to the high effective temperatures $(40,000$ to $70,000 \mathrm{~K})$ in the inner parts of the discs.

The theoretical models were run assuming a boundary layer between the disc and the white dwarf. The effective temperature reaches a maximum in the boundary layer and vanishes at the surface of the WD.

The good fit in the UV range of $\lambda=1000-3000 \AA$ is amazing. What is the source of this good fit? As the effective temperature in the disc increases the maximum of the energy distribution shifts towards lower wavelengths. The opacity below the Lyman edge is very high. The only way to conserve energy is to shift photons from the high frequencies above the Lyman edge to low frequencies below it and thus fill the range above this strong absorption edge. The shortage of space does not allow us to elaborate on this point and to show how the various ionization edges contribute to the total distribution in this range.

Of interest is the very small Balmer jump (almost invisible). When the radiation field is inconsistent with the hydrostatics frequently a large Balmer jump is seen in the theoretical energy distribution (cf. LaDous 1989, Kriz and Hubeny 1987). The observations do not seem to show such a jump.

Figures 3 and $3 a$ are the corresponding graphs for IX Vel. As can be seen from the figure the best fit to the observations is obtained for an accretion rate of $10^{17} \mathrm{~g} / \mathrm{s}$. The quality of the fit can be better appreciated in Fig. 3a where a narrower range of wavelengths is shown (only those for which there are observations) and the scale is greater.

Figure 4 shows the comparison between the prediction of the present model and the observation of the system V3885. Again we find a good fit for an accretion rate of $10^{17} \mathrm{~g} / \mathrm{s}$.

Finally we show the system PHL227. The treatment of the data is the same as before, namely the data is shifted up and down so as to get the best fit to the spectra ignoring the distance of the systems etc. Here the fit is not so good and it is clear that something is missing. Even a trial to change the outer radius of the disc does not improve the agreement significantly.

\section{Discussion}

The most important conclusion from the point of view of the classical novae is the new value for the accretion rates. For all the systems presented here the simple black body fit used so far leads to an overestimate of the accretion rate. If novae have the same accretion rate as novalike variables then there is no problem with the theory of thermonuclear runaways at constant accretion rate. The lower accretion rate eases the situation for the hibernation scenario as well. The reason Prialnik et al. found why there is no runaway for high accretion rates is the heating of the outer layer by the compression of the accreted material. Shaviv and Starrfield (1987) show that when heating from the boundary layer in taken into account the upper limit for the accretion rate to produce a runaway decreases. Hence, a very high accretion rate after a period of low accretion rate may still prevent a runaway by heating of the outer layers. The lower accretion rate found here alleviates this problem if not eliminates it completely. 


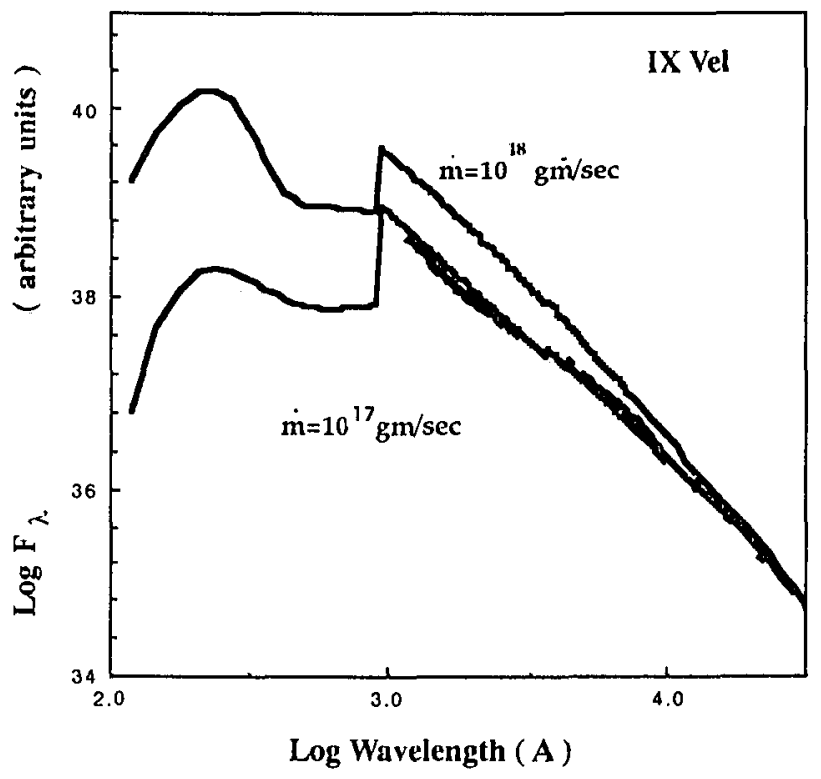

Figure 3. Comparison of the observed energy distribution of IX Vel with the theoretical models for two accretion rates.

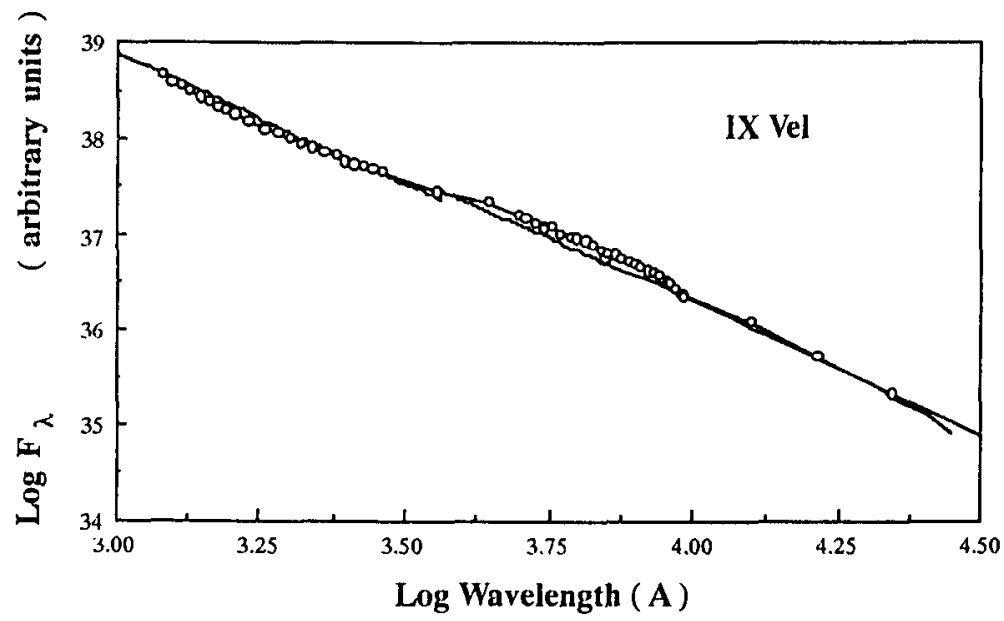

Figure 3a The same as figure 3 but restricted to wavelengths over which there is observational data. 


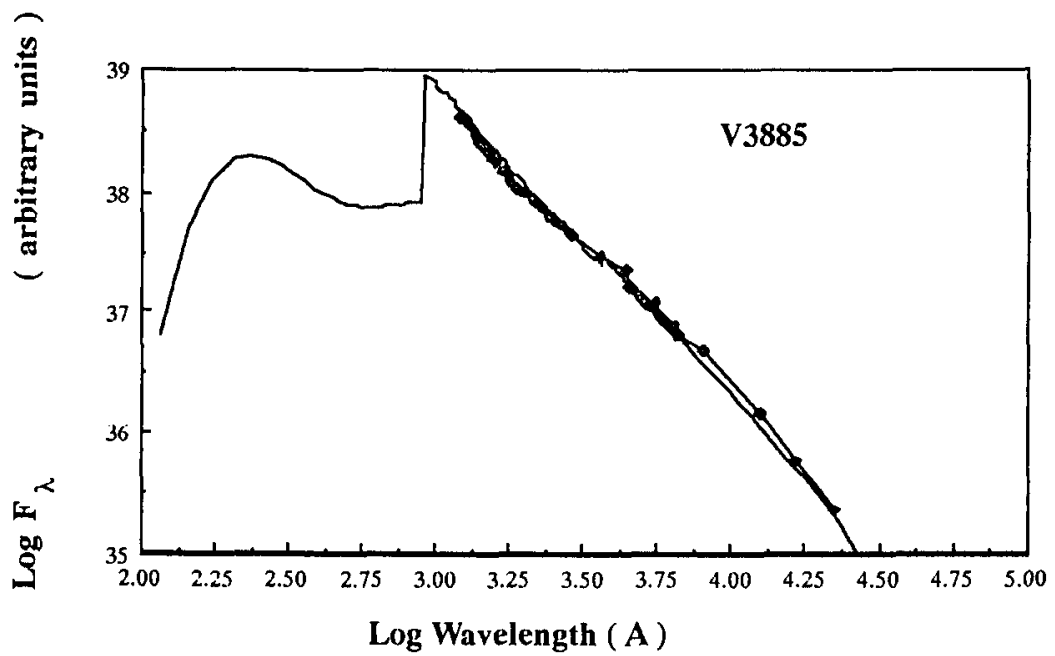

Figure 4 The comparison between the observed and predicted energy distribution for the system V3885. The theoretical model assumes an accretion rate of $10^{17} \mathrm{gm} / \mathrm{scc}$.

The good fit between the theory and the observations is in contrast to the poor performance of simple black bodies. It is imperative to compare the observations with and deduce the parameters of the systems from theoretical models like the one used here. We now discuss briefly the main ingredients of the theoretical model.

\section{Assumptions in the Theory and Properties of the Models}

Our discussion here is restricted to steady state discs. The disc is assumed to posses axial symmetry out to its outer radius. Furthermore, the disc is considered to be geometrically thin. This assumption implies a Keplerian velocity. The matter in the disc loses gradually its angular momentum and moves towards the accreting star. At the same time the extra angular momentum flows outward.

One of the most important problems in the accretion disc theory is the source of the viscosity which operates in the disc and drives the matter into the star. The purpose of this paper is not to discuss this elusive viscosity. We merely point out that: We do not know the exact nature of the viscosity. Once certain assumptions are made about the nature of the viscosity law, models can be constructed and compared with observations of accretion discs. These observations, when examined on the background of theoretical models should enable us to eliminate inadequate theories and restrict possible parameters of the discs. On the other hand, it is important to produce theoretical models under different assumptions about the viscosity and find out how the viscosity affects the radiation that emerges from the accretion discs. 


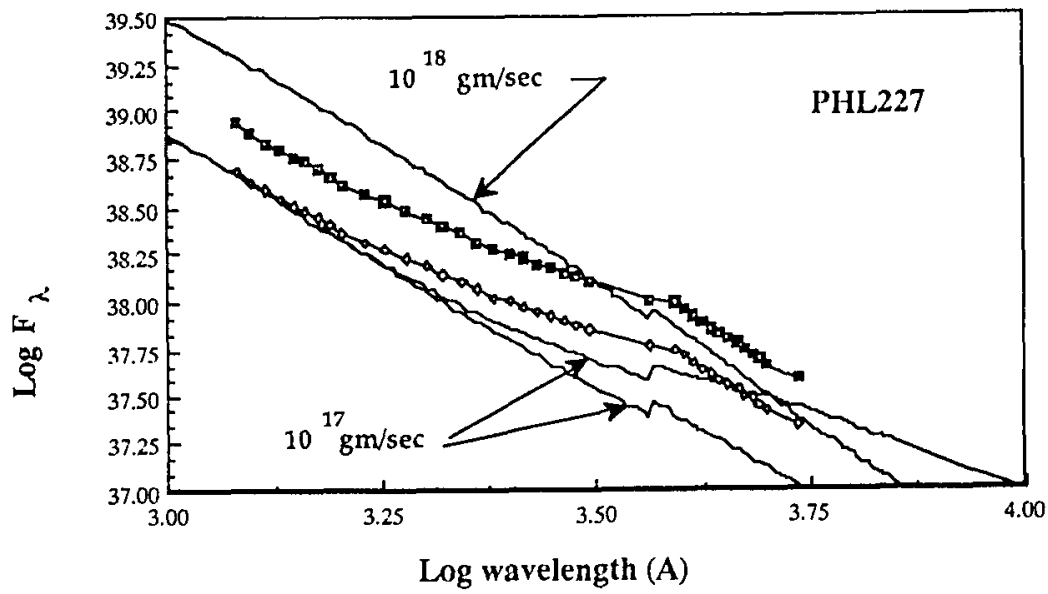

Figure 5. The comparison of the energy distribution observed from the system PHL227 and the theoretical predictions. The comparison is carried out with two accretion rates and two outer radii for the lower accretion rate. The values of the outer radii are 15 and 15 white dwarf radii.The full squares refer to the high accretion rate and the open ones to the low accretion rate.

There are global parameters, like the total luminosity emerging from the disc and the effective temperature of the radiation emerging at a given radius which do not depend on the detailed structure of the disc, the unknown viscosity etc. However, these parameters are frequently found by analyzing only a small part of the spectrum of the object. Without a trustful theory such determinations can be very biased. We did not investigate the possible effects of the viscosity on the predications described here. Here we assume that $\alpha=0.5$ where $\alpha$ is the famous parameters in the $\alpha$-model. The variations of the viscosity can still improve the agreement with the observations. However, we expect the effect of be a small one for discs with high accretion rates.

Since the disc is assumed to be geometrically thin a corollary is the assumption that the largest gradients are in the vertical direction and all the energy released by the matter when crossing radial distance $\Delta R$ flows in the vertical direction only. The radial velocity $V_{r}$ is usually very small compared with the Keplerian velocity at $R$. The energy carried in the radial direction can therefore be neglected in this approximation. The energy released from a ring $\Delta R$ at $\mathrm{R}$ is $\Delta \mathrm{RdL}_{d i s c} / \mathrm{dR}$ and it defines an effective temperature. We have therefore a well defined $\mathrm{T}_{\text {eff }}(\mathrm{R})$ relation.

Several factors combine to make a disc atmosphere so different from that of stars: The run of the gravitational acceleration $g$ with height in the atmosphere is different. In most stars $\mathrm{g}$ stays effectively constant while in red giants it decreases somewhat with height. In discs we find that the gravitional acceleration increases significantly in the region in which the spectrum is formed. The changes of $g$ in the optically thin 
part may be very large (recall that discs are in many cases scattering dominated): The optical depth of the disc is finite. It is in a range in which the boundary condition at the symmetry plane affects the radiation field: The geometry of the disc is neither plane parallel nor spherical. As a consequence the problem of radiative transfer and in particular, the temperature optical depth relation must be derived from a set of assumptions that pertain to the disc.

A large number of observers and theoreticians is interested only in the value af the accretion rate $\dot{m}$ and not in the details of the model. Consequently a common wisdom is that knowing the details of the disc is not mandatory if only $\dot{m}$ is desired. We comment that (a) even for a black body model disc the size of the disc (inner and outer radius) cannot be ignored and (b) since frequently only a small part of the spectrum is observed, some bolometric corrections are required and they can be found only from good and trustful theoretical models. We believe that the comparison with black body results shown previously demonstrates well this point. Hence, even the most fundamental parameter for the disc - the accretion rate - cannot be determined without specifying the structure of the disc. Methods like fitting a single temperature black body or a stellar atmosphere (cf. Wade 1984) are bound to yield dubious results. The number of parameters in any disc model is relatively large and fitting the theory to a small number of data, say 2-3 monochromatic magnitudes, may result in large errors. Since the number of parameters is high a fit over as wide as possible range of wavelengths is imperative if results with high degree of confidence are desired. Obviously the more data is fitted (lines, Balmer jump etc.) the better.

In view of the above discussed situation we solved the radiative transfer problem from a suitable set of assumptions and under the conditions appropriate to the disc and coupled in a self consistent manner to the hydrostatics. The method used is a general one and can be used to check the effect of various assumptions on the energy mechanism, viscous energy dissipation, structure of the boundary layer etc. Here however it is used to deduce the accretion rate in novalike variables.

\section{Method of Solution}

In the problem of the disc the exact height of the photosphere is not known a priori and so is the gravitational acceleration. A change in the height of the photosphere means a change in the gravitational acceleration and with it a change in the entire structure. Hence, we have first to find the location of the photosphere. Next, unlike stellar atmosphere, the flux is not constant throughout the height of the disc. On one hand the energy produced in the optically thin layers is (probably) small because the density and pressure are low, but on the other hand, since these layers are poor radiators (and absorbers) a small energy production in these layers has a large effect on the final temperature these layers will finally settle to in the equilibrium state (Shaviv and Wehrse, 1987). Here we only mention that in some theories for the viscosity in the disc and angular momentum transfer, the energy dissipation is by means of a wind and then the dissipation and energy source is in the very outer layers (Begelman et al. 1983). It is not at all clear how the stresses are transferred in the vertical direction. In any case the radiative transfer must be solved under the supplementary condition at the symmetry axis $z=0$ namely the vanishing of the energy flux in the atmosphere. The 
volume density, the surface density and even the total surface density are not known before the start of the calculations.

In view of the situation described above we approached the problem in the following way. A temperature - optical depth relation is assumed and a height $Z_{0}$ is guessed where the optical depth is sufficiently low. At this point we know the total luminosity from the ring, the effective temperature and with the aid of $T=T(\tau)$ we can integrate the hydrostatic equilibrium from $Z_{0}$ downward to $z=0$. If the height $Z_{0}$ is the correct one, then the flux will vanish at $z=0$. If not, we now iterate for $Z_{0}$ keeping the $T=T(\tau)$ law fixed until the flux vanishes at $z=0$. Once the flux vanishes at the symmetry axis the hydrostatic model is consistent with the energy generation but not with the radiation field. We turn now to the iteration for the radiation field. The iteration for the radiation field is carried out in the following way: the run of the density, pressure, monochromatic opacity etc with height are taken from the hydrostatic calculation and are kept constant during the iteration for the radiative field. Also, the energy produced in each height $\Delta z$ is kept constant. The radiative transport equation is turned now into an equation for the temperature at every height in the disc. When the iterations for the temperature converge we calculate a new $T=T(\tau)$ relation whereby the temperatures are the new ones and the optical depths are still the old ones. If the new $T=T(\tau)$ relation agrees well with the previously used relation, the iteration is stopped and the hydrostatic structure is consistent with the radiative transfer. However, if the two temperature relations do not agree, the whole process of solving for the hydrostatic and then for the radiative field starts again but now with the new $T=T(\tau)$ relation.

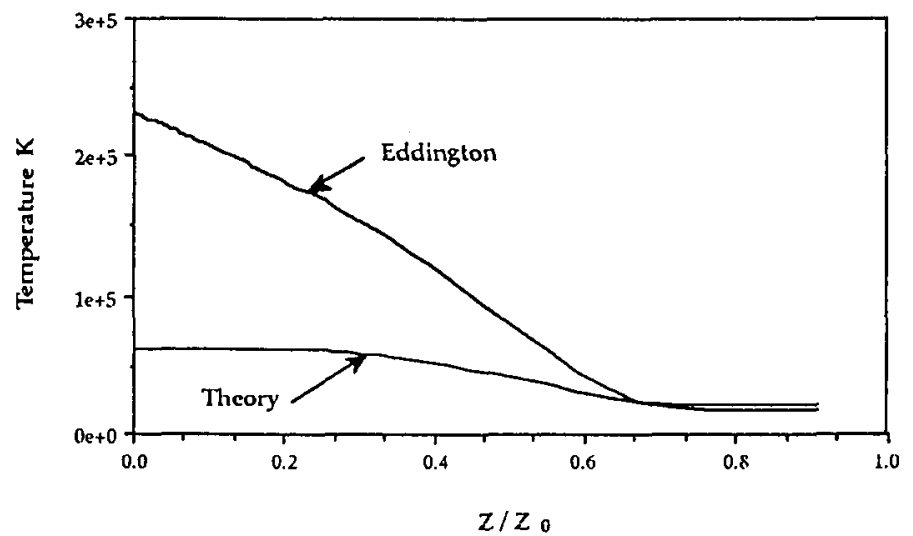

Figure 6 Comparison between the Eddington approximation for the temperature distribution and the temperature distribution found in the theoretical model.with an accretion rate of $10^{18} \mathrm{gm} / \mathrm{sec}$.

A natural question is how bad is the simple temperature distribution and what is the effect on the emerging flux and why bother with the iteration for the temperature which is quite laborious and problematic. In Fig. 6 we show the actual temperature profile found in the iterations and the simple Eddington approximation. The effects on the flux are devastating as can be seen in Fig. 7 where the variations of the flux during 


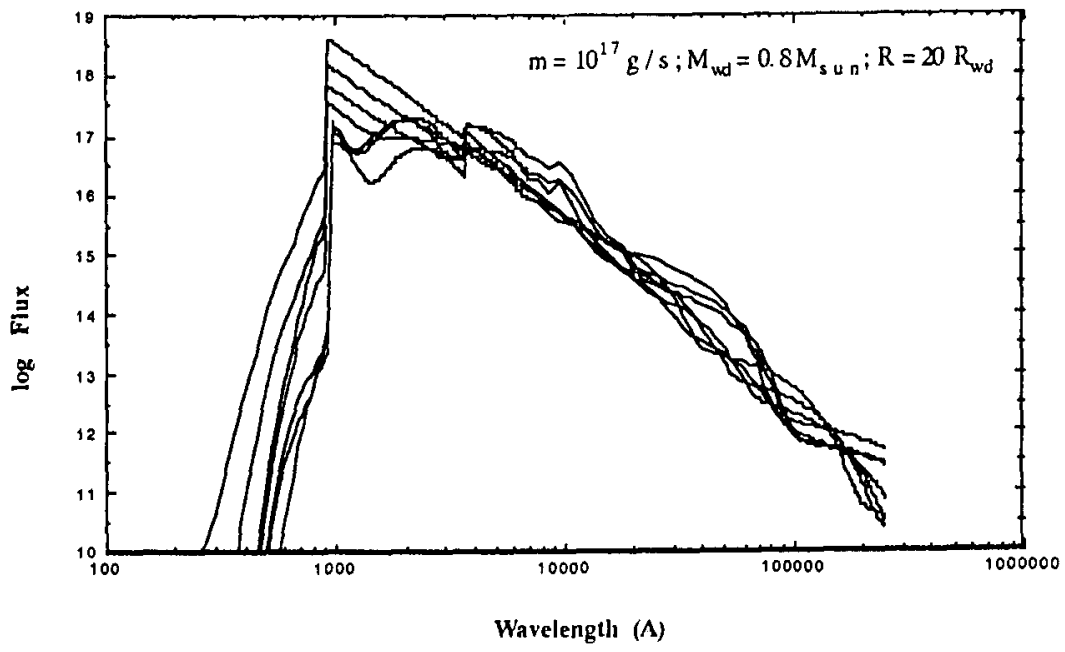

Figure 7 The variation of the energy distribution during the iterations. The variations shown here are for a single radius.

the iterations is shown. The flux is extremely sensitive to the temperature profile, the gravitational acceleration, the height of the photosphere etc. We conclude that proper temperature distribution is required to obtain meaningful results.

Acknowledgement: This research was partly supported by the German-Israeli Foundation for Scientific Research and Development (grant I-94-142.7/88).

\section{REFERENCES}

Bath, G. and Shaviv, G.: 1976, M.N.R.A.S. 175,305

Begelman, M.C., McKee, C.F., and Shields, G.A.: 1983, Astrophys. J. 271,70

Begelman, M.C., McKee, C.F.: 1983, Astrophys. J. 271,89

Greenstein, J.L., and Oke, J.B.: 1982, Astrophys. J. 258,209

Haug, K.: 1987, Astrophys. \& Space Science 130, 91

Haug, K., and Drechsel, H.: 1985, Astron. \& Astrophys. 151, 157

King, A.R., and Shaviv, G.: 1984, Nature 308,519

Kriz, S., and Hubeny, I., 1987, Astrophys. \& Space Science 130, 341

LaDous, C.: 1989, Astron. \& Astrophys. 211, 131

Patterson, J.: 1984, Astrophys. J. Supp. 54, 443

Prialnik, D., Livio, M., Shaviv, G., and Kovetz, A.: 1982, Astrophys. J. 257, 312

Shara, M.M., Livio, M., Moffet, A.F.J., and Orio, M.: 1986, Astrophys. J. 311, 163

Shaviv, G., and Starrfield, S.: 1987, Astrophys. J. Lett. 321, L51

Shaviv, G. and Wehrse, R. 1987, Proc. IAU Coll. \# 96, Davis Philip, A. G., Hayes, D.

S., Liebert, J. W., eds., p. 457

Tylenda, R.: 1977, Acta Astron. 31, 267

Wade, R.A.: 1984, M.N.R.A.S. 208, 381

Wargau, W. Drechsel, H., Rahe, J., and Bruch, A.: 1983, M.N.R.A.S. 204, 35 